Fordham University DigitalResearch@Fordham

1965

\title{
Subjectivity and Objectivity
}

Patrick Heelan

Georgetown University, heelanp@georgetown.edu

Follow this and additional works at: https://fordham.bepress.com/phil_research

\section{Recommended Citation}

Heelan, Patrick, "Subjectivity and Objectivity" (1965). Research Resources. 18.

https://fordham.bepress.com/phil_research/18

This Article is brought to you for free and open access by the Hermeneutic and Phenomenological Philosophies of Science at DigitalResearch@Fordham. It has been accepted for inclusion in Research Resources by an authorized administrator of DigitalResearch@Fordham. For more information, please contact considine@fordham.edu. 
CHAPTER FIVE

\section{SUBJECTIVITY AND OBJECTIVITY}

\section{SECTION I: SUBJECTIVITY AND OBJECTIVITY DEFINED}

\section{Public Obiectivity}

Objectivity, the property of being an object of human knowledge, has many senses. In the first place, it can mean the property of being valid for a general public; its contrary being the subjectivity of the private, individual and incommunicable act. This kind of objectivity, which we call public obiectivity, is necessary for objects of natural science. There are, however, two kinds of public objectivity. One belongs to an idea (or concept), and the other belongs to a reality in its World. The former is that property possessed by an exact and precise definition, nanlely, of being independent of particular places, times and factual occurrences; this belongs not to any World of the real, but to the reahn of ideas. The latter, however, belongs to a shared World of real things. It is the object of factual judgements, founded upon perception and - unlike the precision of an idea - it is accompanied by an irreducible element of impreciseness and indeterminateness.

Public objectivity, as Kant saw it, is based upon the presence of pure synthetic a priori features in our knowledge. Such features are the "axioms of intuition", the "anticipations of experience", the "analogies of experience" and the "postulates of empirical thought in general" 1. Euclidean geometry, causality (in the sense of antecedentconsequent legality between successive phenomena), the permanence of "substance" were universal and necessary aspects of scientific thought because, for Kant, they belonged to the intentionalitystructure of every scientific question. Heisenberg, on the other hand, points out again and again that relativity and quantum mechanics have shown that it is sufficient if the conditions just described be universal

1 1. Kant, Critique of Pure Reason, trans. by Norman Kemp Smith (London, Macmillan, 1963), pp. 194-256. 
only in a well-defined domain and necessary only as a matter of fact. That is, the Kantian synthetic a priori elements of natural science give no more than a possible and hypothetical ground for the construction of a scientific object; a process of empirical testing has to be employed to ascertain whether or not the possible and hypothetical ground is an explanation in fact in this domain and where the boundaries of its domain of applicability are to be found.

Besides the Kantian a priori, there are also other a priori elements which can ground the public objectivity of a scientific object. These are the forms of possible physical theories, mathematical structures for the most part, suggested by empirical data and originating in acts of creative enriching insight. These theories are a priori to experience, not in the sense that they are antecedent to all experience like Kant's Pure Science of Nature, but because they are 'antecedent to the process of empirical testing on the basis of which alone a theory is accepted or rejected. A theory has the structure of an ideal norm composed of a self-defining set of relations. Because the norms are ideal and do not involve acts of perception, these may be shared by a community of scientists who speak the same scientific language, and who can make independent tests of any theory irrespective of particular places and times. The theoretical entities or objects constructed by this process have a comnlon and public value which defines a kind of objectivity which we are tempted to call scientific objectivity, but, since we envisage the problem of such entities within the broader context of being, we prefer to use the term public Objectivity.

In the second place, let us distinguish from one another, several classes of public objects and their noumenal correlates.

\section{Thing}

The first class of public objects is an object which is a unity, identity, whole and the stable subject of properties; it may be either an object given in perception or a constructed object, like an electron, which is linked by us to reality through observable symbols. In either case, the transcendent being correlated with this object, if such exists, is called by us a thing. A subdivision of thing is body.

\section{Body}

The second class of public objects is a phenomenal object, to which corresponds a body (in the strict sense) as its noumenal correlate. A phenomenal object belongs to the class of objects which, as Husserl 


\section{SUBJECTIVITY AND OBJECTIVITY}

said, are "given primordially in perception". It might be described as a stable subject of perceptible properties in a spatially organised World. Allied to the notion of body as the transcendent correlate of a phenomenal-and, therefore, perceptible- object, there are two limiting concepts which we shall include under the name body: they are: (I) whatever is conceived to have determinate spatial coordinates at each instant - as, for example, a classical body or a classical particle - even though it might not be perceptible, and (2) a field which is conceived to be an infinitely extended medium for threedimensional wave motions. These last two classes of objects enter the sphere of reality through their respective observable symbols.

\section{Empirical Objectivity}

The kind of objectivity which is based upon the exteriority of subject and object in perception is given by us the name empirical objectivity. This is divided into phenomenal objectivity for the phenomenal object) and bodily objectivity (for a body). This kind of object, however, is not so constituted by the act of knowing that it is entirely separated from or independent of all subjectivity; exteriority implies its correlate interiority, viz., of a subject. It is then always an objectfor-me.

\section{Formal Objectivity}

While it would evidently be contradictory to state that within the relation of bodily or phenomenal objectivity a subject could simultaneously be the subject and object of knowledge; there is a kind of objectivity in which even the subject can know itself objectively: we call this formal objectivity. This is an objectivity constituted by an affirmation which simply releases it from dependence on a knowing subject as such. It belongs to whatever is affirmed as a virtually unconditioned object on the basis of evidence. In physics, this evidence is provided by a process of testing and verification. This kind of object we call an object in the strict or formal sense; for its intention is simply to express what is, independently of the act whereby I know it as an object-for-me. The noumenal correlate of an object in the strict or formal sense (or a strict object) is an individual existing being, or a relation between individual existing beings.

Although formal objectivity according to its definition is different from both bodily and public objectivity, it is nevertheless implied by both of these types and is in fact an essential element of both. For the 
bodily object which is out there, exterior to me as a bodily subject, is also - except in phenomenalist metaphysics - affirmed to have an existence independently of the relation of exteriority which it has acquired to myself as a knowing subject. The relation of exteriority to a knowing bodily subject is not constitutive of the body-in-itself, but is a relation added to some absolute ground which exists (we may not know how or by what) independently of its phenomenal presentation within the field of my perception. Even pure phenomenalism however cannot escape formal objectivity; for the very positing of phenomenalism as a true philosophy is an act whose sense is to separate a certain mental content from its dependence on my or any subjectivity. This mental content is an object to which the relation of bodily exteriority simply does not apply: it has, however, public objectivity.

Public objectivity also implies formal objectivity and contains it within itself. There are two kinds of public objectivity: of an idea and of a reality in its World. We are here concerned with ideas only in so far as they are asserted or affirmed of something given in experience and hence as a property of a real situation within a World. An idea however may state the relation of a thing to a knowing subject or a relation existing between things. In either case, the terms of the relation are presupposed by the relation and posited in some way to exist independently of their relation to my subjectivity; that is, the relation to my subjectivity is merely the means through which some absolute ground makes itself present to me in my experience. This is very evident in the case of ideas which, like physical properties, express thing-to-thing relations; for even, if it should be argued that the relation generates its own terms, neither of the terms of a thing-to-thing relation is (in physics) a knowing subject and hence the positing of such a relation satisfies the definition of formal objectivity in a special way.

These considerations however suggest an important question: Is formal objectivity to be atrributed equally to every aspect of the public object? Or are there aspects to which public objectivity can be correctly attributed but which lack nevertheless formal objectivity? Let us recall that it is sufficient for a public object merely that it be understood, recognised and described by all in the same way. Consequently, public objectivity does not require that a priori subjective elements, if there are any, which are common to a certain way of knowing but are nevertheless extrinsic to that which is formally and strictly affirmed, should be consciously distinguished from the content of that which is strictly affirmed. These a priori elements of the public 


\section{SUBJECTIVITY AND OBJECTIVITY}

object belong to the intentionality-structure of the knowing in so far as this is a human method of doing scientific research, and they constitute the matrix in which the strict object makes its appearance. The disengagement of the strict object from its setting of scientific methodology is, we believe, one of the principal epistemological problems of quantum mechanics, and we reserve this problem for section III of the present chapter.

\section{Reality and its Criterion}

Let us distinguish, moreover, the meaning of the term "reality" from the criterion of reality with reference to a certain knower. The former defines what is meant by the term. The latter is that on account of which a thing is said by a certain knower to be real: in our case, it is the sign through which its reality is manifested to us. In the rationalist intentionality-structure of classical physics, "reality" means "a body in the strict sense (but with idealised boundaries and coordinates), or whatever agrees with the limiting concepts of classical particle or classical field". Its criterion is the appearance in experience of an appropriate - if vague - indication of its presence such as, e.g., the recording of a non-vanishing field intensity. In the empiricist view, «reality" means "whatever is here and now perceived as a body in the strict sense". For it, the meaning and the criterion of reality are identified. Anticipating a later section, let us state here our own view for the sake of completeness: (1) the criterion of physical reality in regard of a human knower is not identical with its meaning, since we have no intellectual intuition of physical reality; (2) the meaning of "reality" is "whatever is defined by the object in the formal sense", while (3) its criterion is a manifestation of its presence through observable symbols, and critically judged to be such, within a World of real things.

\section{Subjectivity}

Just as there are many kinds of objectivity, so there are many kinds of subjectivity. This we define to be the absence of a corresponding kind of objectivity. There is then (i) a subjectivity which is an absence of empirical objectivity. This may be either the type of interiority which is the strict correlate of the exteriority of an empirical object, or simply a lack of bodily objectivity. The latter is one of the senses in which the quantum mechanical system is said to be non-objective. (ii) There is subjectivity which is an absence of public objectivity. This is the sub- 
jectivity of unshared or incommunicable private experience or observation. This too is said to be a new and inescapable factor of quantum mechanical science. Finally, (iii) there is a subjectivity which is the absence of sufficient evidence for the virtually unconditioned affirmation of a strict object. This is the subjectivity attached to a mere supposition or hypothesis.

\section{SECTION II: EMPIRICAL OBJECTIVITY}

\section{Objectivity and Exteriority}

Quantum mechanics denies that an atomic physical system is objectifiable as a body; i.e., it says that a quantum mechanical system does not possess precisely determinable space-time coordinates and momenta independently of particular acts of measurement and observation. The denial in question refers to the public empirical objectivity of a body. This is a direct consequence of the Principle of Complementarity. "Science", as Heisenberg said, "no longer confronts nature as an objective observer" 1 ; the objects of science are not bodies, existing out there in isolated exteriority to the knowing subject. From this denial of the exteriority of the object, Heisenberg went to the opposite extreme and concluded that the object was really an interior act: "The object of research is no longer nature itself, but man's investigation of nature. Here... man confronts himself alone" 2.

The failure of an objectivity founded upon exteriority alone, however, does not entail as a necessary consequence the kind of interiority in which "man confronts himself alone"; for there is a third possibility, viz., a more discriminating critique of the subject-object relation in science, and a better analysis of the structure of the scientific object in the strict or formal sense 3.

A more careful analysis of the subject-object relations shows that

1 Heisenberg, Physicist's Conception of Nature, p. 29.

2 Ibid., p. 24.

3 That the principal kind of objectivity envisaged by most physicists is one based upon the relation of exteriority, is illustrated by von Neumann's account of the measuring process (Mathematical Foundations of Quantum Mechanics, chap. VI, especially pp. 420-421). Here he uses the principle of psycho-physical parallelism to establish the distinction and relation between subject and object in the measuring process; the division between the two, in his account, is evidently a spatial division, which he called the Schnitt or boundary. "That the boundary can be pushed arbitrarily deeply into the interior of the actual observer is the content of the principle of psycho-physical parallelism..., but this does not change the fact that in each method of description the boundary must be put somewhere" (my italics), ibid., p. 420. Heisenberg paraphrases this passage in Niels Bohr etc., p. 27. 


\section{SUBJECTIVITY AND OBJECTIVITY}

there are many different kinds based upon a variety of different differentiating relations. The exteriority of a body vis-à-vis a human knower as a bodily subject is not the only kind of objectivity which a scientific object can have. In fact, it would be a serious misunderstanding of scientific method to state that objectivity of this kind belongs essentially to a physical system as known. This was one of the errors of the intentionality-structure of classical physics.

Empirical objectivity belongs to a unity, identity whole which is perceived as such; a physical system, however, is a thing, i.e., a unity, identity whole which is the subject of physical properties. We have already shown that physical properties are not defined relative to perception, but relative to a self-correlated set of interactions between things. It follows from this that neither a physical property nor a physical system contains in its definition anything that relates it intrinsically to elements of perception. Comparing the definition of a physical system with that of a body, we see that the former in no way implies - though it does not exclude - that it be a body. What actually is perceived by a scientist is a complex of bodies and bodily properties which comprise the measuring apparatus. This is the context in which the observable symbol occurs, which is the criterion that manifests to us the reality of a quantum mechanical system. That the physical system should itself be one of the bodies of this complex is not required by the physical theory.

The failure of empirical objectivity for microscopic systems, then, is not only understandable, but it might well have been anticipated by a more careful analysis of scientific method. We do not mean to say that Heisenberg's Indeterminacy Relations should have been predicted on the basis of an analysis of scientific method; for this is founded upon new empirical data; but that the rearguard action in favour of theories of a "classical sort" should have been dropped long ago, and for reasons based upon the logic of scientific method.

\section{SECTION III: PUBLIC OBJECTIVITY}

\section{Heisenberg and Public Objectivity}

Public objectivity, as we have seen, is a necessary condition of science; since, without it, there could be no scientific community, no scientific language and no collaboration towards well-defined goals. It is surprising, then, that many physicists should find that the 
possibility of this kind of objectivity is restricted by the fonnal structure of quantum mechanics.

Heisenberg never doubted the public objectivity of quantum mechanics. It was for him one of the indubitable facts which were the starting point of his philosophy. "The physicist", he wrote, "must postulate in his science that he is studying a world which he hinlself has not made, and which would be present, essentially unchanged, if he were not there" 1 . The critical problem in the philosophy of science, as Heisenberg saw it, was to express the a priori conditions of possibility -both subjective and objective- of a science which, like quantum mechanics, contradicted so many of the universal and necessary conditions accepted both as a part of pure science and as a part of philosophy since the time of Kant.

The contrast between the public objectivity of quantum physics as a science and the "subjective element" in the acts which comprise the exercise of this science, is brought out in many parts of Heisenberg's writings. "The objective reality of the elementary particle has been strangely dispersed", he wrote, "not into the fog of some ill-defined or still unexplained conception of reality, but into the transparent clarity of a mathematics which no longer describes the behaviour of elementary particles, but only our knowledge of their behaviour" 2. Again in his celebrated article contributed to the collection, Niels Bohr and the Development of Physics (1955), he wrote: "This representation [of a closed system by a ray in Hilbert space] ... is completely 'objective', i.e., it no longer contains features connected with the observer's knowledge, but it is also completely abstract and incomprehensible since the mathematical expression PHI(q), PHI(p), etc., do not refer to real space or to a real property, it thus, so to speak, contains no physics at all" 3. Such a description is "complete and objective" but "not real". To make this a "description of Nature" it has to be linked to the question of "how real or possible experiments will result". The interaction of the system with the measuring apparatus is described mathematically by a mixture and "thus the description contains, besides its objective features, ... information about the extent of the observer's knowledge of the system" 4. This latter, he calls, the "subjective element" in our knowledge of nature; since a mixture gives only "incomplete information" about the actual state of the

1 Heisenberg, Niels Bohr etc., p. 24.

2 Heisenberg, Physicist's Conception of Nature, p. 15.

3 Heisenberg, Niels Bohr etc., pp. 26-27.

4 Ibid. 
object. The "subjective element" in question belongs to the sphere of the private experience of the observer.

\section{The "Subjective Element"}

For Heisenberg, the ((subjective element" in quantum mechanics is twofold: (1) the failure of empirical objectivity (in the sense of classical physics) for quantum mechanical systems, and (2) the failure of public objectivity in quantum mechanics resulting in an insurmountable subjective barrier limiting public knowledge of atomic systems to irreducibly probabilistic laws.

The failure of empirical objectivity led to a rejection of the rationalist view of reality. One would think that Heisenberg, like Bohr, swung to the other extreme, of outright empiricism. This is ipdeed the impression one receives - for example, from his insistence that reality is encountered only in what is actually experienced, Le., in "observation events", and that the true description of reality is always of happenings in three-dimensional space. His original insight on the importance of observables in physics contained, as we have already shown, a strong empirical element. However, Heisenberg, the theoretical physicist, had a strong rationalist bias and this led him eventually to an explicit rejection of empiricism. Being persuaded of the rationality of nature, he saw in Berkeley and Locke an abdication of the power of reason 1. We shall examine in another chapter the attempts he made to overcome this twofold crisis 2.

The outcome of this tension in Heisenberg was a synthesis of the two dialectical extremes by means of a Kantian distinction between an empirical reality represented by the phenomenal object and a noumenal reality or thing-in-itself, which would be the content of an intellectual intuition of physical reality, if we had such an intuition. However, Heisenberg surmises that we have no such intellectial intuition and consequently that reality in the sense of thing-in-itself is formally unknowable by us. Nevertheless, we are not deprived of all contact with noumenal reality, since human reason and empirical intuition preserve a symbolic vestige of it in so far as they are related by their activity to an unspecified noumenal correlate. Rationalism and empiricism are both rejected but ultimately reconciled on the deeper level of the knowing subject where they express different aspects of the dynamic structure of human intentionality. The outcome of this is to divide the 
meaning of "reality" into two: a phenomenal reality defined as the object of categorised empirical intuition, and a noumenal reality defined as the object of a kind of intellectual intuition which we do not possess.

Originally, for Heisenberg, these were the only objective meanings of reality. The quantum mechanical system, however, does not fulfil either meaning. Although every observation-event of a quantum mechanical system terminates in the empirical realities of the measuring instrument and its response, no well-defined permanent and causally-related phenomenal object can be formed of the quantum mechanical system and, 'consequently - assuming parallelism - no well-defined noumenal correlate exists. The failure of the quantum mechanical system to satisfy either of the two definitions of "reality", was for a long time blamed by Heisenberg on a radical insufficiency of the human knowing subject. Knowing neither the empirical nor the noumenal reality of the quantum mechanical system, the human knower was forced to represent it by an artificially constructed idea, viz., the wave function, which "contains no physics at all" 1. In later years, Heisenberg proposed a third and new meaning for the term "reality", to which he gave the names "potentia", "objective tendency", or "objective possibility". This was neither a pure idea, nor an actual event (an empirical object), but it was a real possibility of producing ideal frequencies 2.

The root cause of Heisenberg's perplexity is to be found in his inadequate theory of knowledge and scientific method, and particularly in an underlying parallelism which assumes that the reality of a physical system or property is either the direct object of an act of observation (a phenomenal reality) or something directly correlated with it in a parallelistic sense (a body or the kind of bodily property which is founded upon a thing-to-us relation). If this is assumed, then, the quantum mechanical system, to which no stable and coherent empirical object corresponds, is not real in either of the two senses discussed. The direct object of an act of observation is not, however, as we have shown, the reality of the physical system. 'It is merely its observable symbol. The lack of coherence of a set of sensible symbols (in this case, of two mutually exclusive sets, namely, discrete or particle symbols and continuous or field symbols) does not imply a lack of coherence in the thing symbolised. Moreover, the connection between the observable symbol and reality is not one 
of parallelism in the usual sense for, if this were so, reality would be as incoherent as the symbol; its relation to reality is far more complicated and will be discussed in chapter IX.

As for the irreducibly probabilistic nature of quantum mechanical laws, we have stated our view that this does not imply such an incursion of private subjectivity that our power of knowing reality suffers an essential check at the quantum level. Probabilistic laws do not spring from ignorance nor from the inescapable perturbation of an object by an observer-subject, but they arise as a necessary consequence of our abstractive mode of knowing individual and concrete things. Only an ideal norm can be defined precisely by the human mind. This always has the structure of a law from which concrete cases diverge only randomly. Random deviations lead to statistical laws. Hence, quantum mechanics, in which we find the organic union of both statistical and deterministic laws, expresses the most general form in which a scientific theory can be expressed by a human investigator. We shall return to these points later on.

\section{Problem of Public Objectivity in Quantum Mechanics}

For many physicists, however, the logic of quantum mechanics leads to the conclusion that quantum mechanics is concerned with the private act of observation of a scientific observer and that, moreover, this private act is capable of destroying previously existing correlations and so of effecting public and observable changes in the course of nature. Under these circumstances, the public objectivity of science is put in jeopardy.

To illustrate this problem, we shall use an example adapted from Heisenberg, changing the conditions a little in order to bring out the salient points better, but following the line of Heisenberg's argument 1.

In our exposition, we shall keep as close as possible to Heisenberg's language, thought and manner of interpreting the experiment. In the next sub-section, we shall analyse more fully the views "expressed. Let us consider a beam of atoms all prepared in the state " $\mathrm{m}$ " (for example, of magnetic moment $\mathrm{m}$ ) which is passed in succession through two inhomogeneous fields $\mathrm{F}_{1}$ and $\mathrm{F}_{2}$. Transitions take place during the passage through each field. The two fields are accompanied by separating fields which separate the different states into different beams so that an observation made on the relative position of the atom by an observer is equivalent to an observation of state (see figure).

1 Heisenberg, Physical Principles etc., pp. 59-62. 


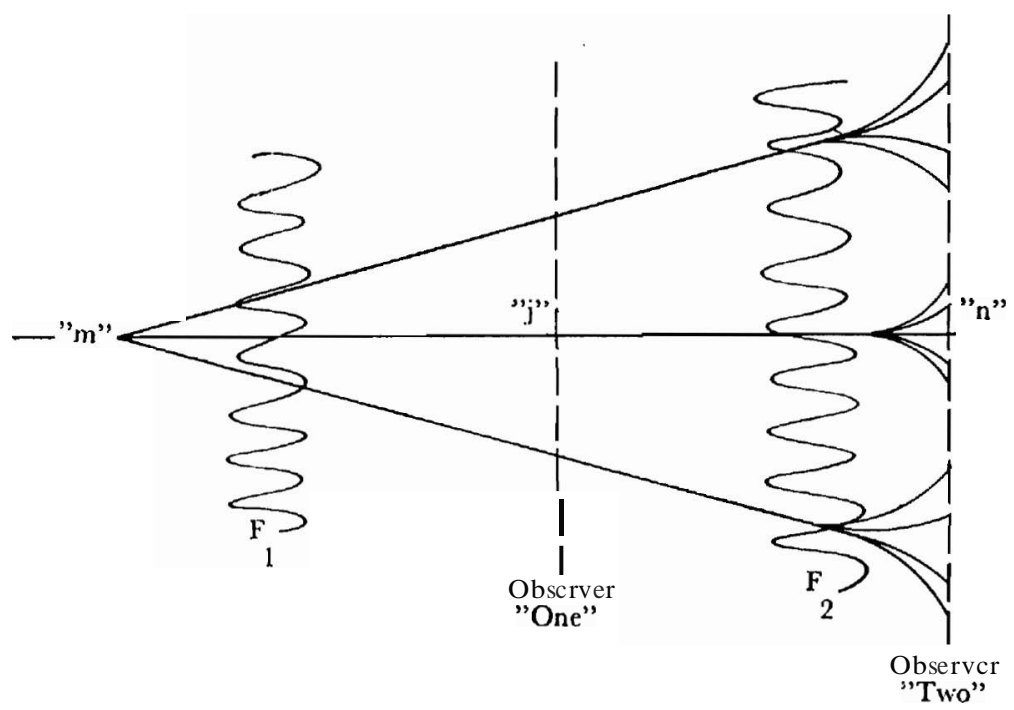

There is an observer (observer "One") placed at an intennediate position between $\mathrm{F}_{1}$ and $\mathrm{F}_{2}$; and a second observer (observer "Two") placed on the far side of F2. Each observer has an apparatus designed to measure an atom's position, which, for him, is an index of the magnetic moment of the passing atom.

Each act of observation has two moments: an exchange of at least one photon with the atom, and a conscious act taking cognizance of the result. We call the whole act, the act of observation, and the latter part, the conscious act 01 observation. The action of the separating fields with the act of observation constitute the lull act of measurement.

According to Heisenberg, three cases occur: - Case I: Observer "One" does not make an observation. The entire experimental set-up is then considered to be an isolated system and consequently to be in possession of a pure case wave function 1. The transition probability from state " $m$ " to state " $n$ " in the experiment in this case is:

$$
\sum_{j}\left|S_{m j} S^{*}{ }_{j n}\right|^{2}
$$

where $S m j$ and $S^{*}$ jn are the matrix elements for transitions during the passage of $F_{1}$ and $F_{2}$ respectively.

We shall discuss below why it is significant for the result (I) that the observer "One" avoid interaction with the passing atom.

Case II: Observer "One" allows his apparatus to interact with the passing atom but fails to make a conscious act of observation. The system splits into two independent systems, annihilating the interference of probability waves, and the transition probability from " $\mathrm{m} "$ 
to " $n$ " becomes in this case:

$$
\sum_{\mathbf{j}}\left|\mathrm{S}_{\mathrm{mj}}\right|^{2}\left|\mathrm{~S}^{*}{ }_{\mathrm{jn}}\right|^{2}
$$

A mixture is formed when the apparatus is separated from the measured object, and all correlations except the one-to-one correlation between the states of the apparatus and the states of the object are destroyed.

We shall discuss below whether and in what sense we can speak of the transformation of a pure case into a mixture apart from the two stages in the act of observation, or apart from the communication of the result to others.

Case III: Observer "One" observes that the state of the passing atom is " $\mathrm{j}$ ", and communicates his knowledge. The total transition probability from state " $m$ " to state " $n$ " is in this case:

$$
\left|S^{*}{ }_{j n}\right|^{2}
$$

The difference in form between (I) and (II) shows that some difference exists between Case I and Case II. The difference is in the transformation of the pure case into a mixture at the intermediate position in Case II. Heisenberg implies that the transformation from the pure case into a mixture is a physical effect - like the filtering of a liquid - and is produced by the "partly undefined interactions of the measuring apparatus" 1 . He assumes that the difference in Case II is prior to the conscious act of observation. It is clear that he wishes thereby to save the public objectivity of quantum mechanics. Note that Heisenberg regards the "reduction of the wave packet" - which is the final result of the measuring-process - as occurring in two steps: (1) the formation of the mixture, which is prior to the act of observation, and (2) the act of observation which ascertains or "registers" which of the possible states has been actualised 2. We shall be concerned in the next sub-section with the question, in what sense the formation of the mixture is a physical process like the filtering of a liquid, and in what sense it is dependent on whether or not the observer-scientist makes a conscious act before or after the measurement.

1 Heisenberg, Niels Bohr etc., p. 23; also Physical Principles etc., p. 60.

2 Commenting on Einstein's thought-experiment of the semi-transparent mirror, Heisenberg says that "the experiment at the position of the reflected wave packet, exerts a kind of action (reduction of the wave packet) at the distant point occupied by the transmitted packet, and one sees that this act is propagated with a velocity greater than that of light", Physical Principles etc., p. 39. 
in which the observer-subject interacts with the object and undergoes a prise de conscience of this experience. If this interpretation is to be consistent, then the point of division (or Schnitt) on the connected chain of physical processes which unites the object to the observing subject must be capable of being displaced arbitrarily. Whether this point lies outside the body of the observer-subject or inside it should be immaterial to the physical result 1 .

Logically implied in Reisenberg's view of the measuring process is the position that the behaviour and pattern of objects in human empirical consciousness are also subject to quantum mechanical laws. Acts are specified by their objects. If then the object of empirical consciousness is identical with reality, and if reality is subject to the quantum theory, then the behaviour and pattern of objects in human empirical consciousness is also subject to quantum mechanical laws. The quantum theory then takes on the character of a universal explanation for physical and mental events 2. Von Neumann is quite explicit on this point: "It is a fundamental requirement of the scientific viewpoint - the so-called principle of psycho-physical parallelism - that it must be possible so to detennine the extraphysical process of the subjective perception as if it were in reality in the physical world" 3 .

Returning to the difference between Case I and Case II: let us state the dilemma in the following way 4. If observer "One" makes an observation but fails to communicate its result, what, he might ask, is the correct state of the passing atom? Observer "Two" is in a quandary. He does not know whether to treat the case as (a) a pure case of the total isolated system (including observer "One" in the isolated system), or (b) whether to suppose that a mixture was fonned at the intermediate stage. The orthodox physicist says that the latter (b) is the correct alternative. However, if observer "One" were replaced by a non-conscious piece of apparatus, then the orthodox physicist would say that the former (a) is the correct solution. Wigner says of this dilemma: "The argument for the difference in the roles of inanimate observation tools and observers with a consciousness - hence for a

1 Heisenberg, Niels Bohr etc., p. 27: also von Neumann, Mathematical Foundations etc., pp. 418-420.

2 Cf. E. P. Wigner, "Remarks on the Mind-Body Problem", The Scientist Speculates, pp. 284-301: Abner Shimony, Am. Jour. Phys., XXXI (1963), pp. 755-773.

3 Von Neumann, Mathematical Foundations etc., pp. 418-419.

$4 \mathrm{We}$ are adapting for the purposes of our example Wigner's treatment of a similar problem and discussed by him in his article referred to in note 2 above. Cf. also, "Theorie der quantenmechanischen Messung", Physikertagung, Wien, I96I (Mosbach/Baden: 1962), p. 1; "The Possibility of a Self-reproducing Unit", in The Logic of Personal Knowledge (Glencoe, III: 1961), p. 231. 
violation of physical laws where consciousness plays a role - is entirely cogent so long as one accepts the tenets of orthodox quantum mechanics in all their consequences" 1 . Wigner then concludes (1) that Mind is not subject to quantum mechanical laws (This is a significant break with parallelism!); (2) that the Mind can influence the course of nature by its acts, and (3) that the linear mathematical equations of the quantum theory are unsatisfactory 2 .

It has often been pointed out that the epistemology of the "orthodox" interpretation leads to solipsism; for if the private act of observation specifies reality by actualising its potentialities and suppressing correlations, then the only possible explanations of the public objectivity of science are a pre-established hannony between minds, or that there is just one subject identical with all subjects. The first solution raises the problem of how the harmony is pre-established, e.g., by causal influences between different 'subjects, or by the possession of similar a priori fonns of knowledge, etc. ${ }^{3}$. Reasons of simplicity and economy have commended at least in theory the second solution, viz., solipsism, to many physicists 4 .

These are conclusions to which many quantum physicists have come not without a considerable amount of uneasiness 5. The increasing volume of literature on the subject witnesses, if not always to these misgivings, at least to the feeling that the foundations of quantum mechanics needs some maintenance and repair 6. Some physicists, like Ludwig in his most recent paper 7 , think that the validity of quantum

1 Wigner, The Scientist Speculates, p. 294

2 Ibid., pp. 294-298.

3 For example, P. A. Moldauer writes: "The way in which the mind reacts to information about the physical world - and hence the structure of the wave function and of physical theory in general - would have to be regarded as strongly conditioned by the evolutionary, cultural and perhaps personal factors which strongly influence the structure of the human mind", Am. Jour. Phys., XXXII (1964), p. 172.

4 For example, Wigner, The Scientist Speculates, p. 290; and the remarks of the general editor pp. 301-302; also Abner Shimony, lac. cit.

5 E. Schrödinger was the first to draw attention to this aspect of the "orthodox" theory of measurement in "Die gegenwärtige Lage in der Quantenmechanik",, Naturwissen., XXIII (1925), pp. 807-812. Another disturbing consequence of the quantum theory of measurement is the fact that in the absence of observations, the total entropy of the system does not change; it changes however discontinuously after an observation.

6 For example, besides the articles referred to in notes we might mention the following important articles: P. K. Feyerabend, "Problems in Microphysics", in Frontiers at Science and Philosophy ed. by R. G. Colodny and C. G. Hempel (London, Allen and Unwin, 1964); H. Margenau, "Measurements and Quantum States", Phil. Sci., xxx (1963), 1-16, 138-157; H. Margenau and R. N. Hill, Progr. Theor. Phys., Xxvi (1961), p. 727; Y. Abaronov and D. Bohm, Phys. Rev., CXXII (1961), p. 1649; A. Lande, Zeit.f. Physik, CLXII (1961), pp. 410, 558; the articles by Lande, Teller, Born, Bopp and Ludwig in Werner Heisenberg und die Physik unserer Zeit (Braunschweig: 1961).

7 G. Ludwig, Werner Heisenberg usw., pp. 150-181. 
mechanical laws should be restricted to the microscopic domain. Others, among whom are Heisenberg and Wigner, defend its universal validity and draw, what to them is the logical consequence, an immanentist philosophy.

\section{Solution 01 the Problem}

To escape the immanentist tendencies for which Heisenberg and many physicists claim to find support in the structure of quantum mechanics itself, we shall offer our own solution of the key problem placed by the difference between Case I and Case II.

It belongs to the scientist to choose the problem he wishes to consider by choosing the subject matter of the problem. This is an act of the scientist which precedes all the other acts - whether of calculation or of measurement or of observation. It is the bringing to bear of a noetic intention on something that is given or to be given in experience, namely, publicly communicable data. These constitute the subject matter of the problem, and are specified by the experimental procedures or contexts appropriate to the case under consideration. The difference in form between formula (I) and formula (II) indicates that different ensembles of data are described by the two formulae, and different experimental contexts are envisaged. The problem lies in interpreting where, among the conditions listed in the text, or presupposed by these, the difference lies.

According to the Heisenberg of the Physical Principles of the Quantum Theory, the difference is due to the conversion of the pure case into a mixture at the intermediate position, and is caused by the undefined interactions of the apparatus of observer "One" without, however, observer "One" making a conscious act of observation. According to Wigner, it is due to the conscious act of observation.

Neither of these solutions is satisfactory. In the first case, the theory predicts that, although the intermediate interactions are undefined, and although their effect can be reduced to vanishing point by allowing the beams to separate, the correlations characteristic of the pure case will continue unless an observation is made. In the second case, it is difficult to see how the merely noetic act of becoming aware of a particular state of the apparatus can suppress, as Wigner believes, a physical link (expressed by the superposition correlations) operative in nature.

The answer probably lies somewhere between the two views just criticised. The conversion from a pure case to a mixture is not a 
physical change in nature but a logical operation and results from the choice to consider one kind of problem rather than another. It is thus logically prior to all other acts, whether of calculation, measurement or observation. Still in the logical order, this choice is followed by the calculation of the mixture from the mathenlatical equations of the appropriate pure case. The corresponding activities in the experimental order are the erection of or attention to appropriate apparatus: in Case I for observer "Two" alone, and in Case II for both observers "One" and "Two".

Consider now the two cases separately. In case I, the ensemble of data is composed entirely of the results recorded by observer "Two". In Case II, there are two independent subensembles of data; one set recorded by observer "One" and the other set recorded by observer "Two". Heisenberg has stated in the conditions governing Case II that observer "One" does not record his observation but merely lets his apparatus interact with the passing atoms 1. This protocol confuses the issue, since it is clear that the individual terms in formula (II) refer to the observations which observer "One" must be in a position to make and to communicate. In fact, each transition probability is an ideal frequency from which no finite recorded sample diverges systematically. The use of ideal rather than actual (counted) frequencies for the intermediate transition probabilities presupposes that if the actual results were considered (in a finite sample) only random deviations would be noted. It is in this trust in the essential validity of the ideal intermediate transition probabilities which is presupposed by the protocol. It is only accidental that, in the kind of problem here proposed, conscious acts of observation by observer "One" can be omitted without changing the physics of the case. The physical interaction involving an exchange of photons between the apparatus of observer "One" and the passing atoms seems, however, to be essential to the problem and explains the physical and experimental difference between Case I and Case II.

We have already pointed out that, even if the beams corresponding to the different states are separated by $\mathrm{F}_{1}$, the pure case correlation remains, and this is responsible for the wave-like interference of the beams with one another. The greater the separation, however, the less the wave-like interference; and the less the separation the greater the wave-like interference. For, on the one hand, the greater the separation, the less energetic the exchange photon needed to establish the actual

1 Heisenberg, Physical Principles etc., p. 61. 
presence of an atom in a beam, and the less the consequent momentum disturbance of the atom - for the breadth of each beam is supposed to be considerable. And, on the other hand, the less the separation of the beam, the more energetic the exchange photon needed to establish the actual presence of an atom in a beam, and the greater the consequent momentum disturbance of the passing atom. It is just this correlation between the energy of the photon required to count the actual presence of atoms in a beam and the separation of the beams which is responsible for the physical and experimental difference between the two ensembles.

Physical perturbations occurring within the act of measurement are seen to be of two kinds. One is essential to the definition of a physical property; in the example we have chosen this corresponds to the perturbation which separates the states into different beams. It defines the manner for comparing atoms - not according to their resemblances with respect to our direct experience of them - but according to the way they behave within a controlled ensemble of physical interactions. The second kind of perturbation is that due to the act of observation which involves the exchange of at least one photon with the atonlic system. It is this latter perturbation which is not accounted for in classical physics. It is, of course, present in every concrete observation but classical physics is not usually concerned with it. Nor is quantum mechanics particularly concerned with it, since, as we have shown, it can under favorable circumstances be reduced to, an arbitrarily small amount - provided that the states to be distinguished are not continuous. The presence of this kind of perturbation at an intermediate stage in an atomic process can, however, change the statistical correlations between the initial conditions and the final results; this is a new and non-classical property.

In conclusion: we have shown in reply to (the early) Heisenberg that the formation of a mixture from a pure case is not produced by the physical separation of states; and in reply to Wigner, that the formation of the mixture is a logical step prior to all concrete acts of observation. We conclude then that the pure case wave function is merely a mathematical instrument with which to calculate the particular mixture appropriate to the kinds of observations envisaged by the experiment to be performed. From the arguments we have just given, we deduce that quantum mechanics shares to the full the public objectivity of science. 


\section{Wave Function}

What then is the pure case wave function? It is, in the first place, a mathematical instrunlent of a higher logical order of abstraction than a mixture; that is, while a mixture is an ensemble of ideal frequencies of occurrence of values in random samples, the wave function itself represents a potential ensemble of such mixtures, one for every possible final experimental context which could be chosen. The appropriate mixture can be calculated only when the final experimental context is chosen. In the light of this interpretation the saying of Heisenberg that modern physics no longer refers to nature but to our knowledge of nature takes on a new significance 1 . If the object of quantum mechanics is taken to be the wave function (or the physical state as represented by a ray in Hilbert space) then this indeed is a logical entity, i.e., a mere instrument of our knowledge of nature; it is not a part of nature itself. Moreover, rejecting classical parallelism in knowledge we reject also its consequence - the notion that the wave function in configuration space or even the three-dimensional wave packet is the real object of quantum mechanics. The real object of quantum mechanics is its strict object. This makes its appearance, however, within the matrix of scientific method and theory and it has to be disengaged with great care if its true visage is to be seen. It will be the task of the next section to perform this operation.

In the second place, the wave function is connected directly with the experimental context in which the physical state is prepared. In a time independent system, the physical state retains its direct reference to this preparatory experimental context, i.e., it remains an eigen state of this context 2. In the general case, however, the original state undergoes an evolution in accordance with its Schrödinger equation. Its physical state changes deterministically in time. At any instant, its state could be considered to be the eigen state of some (changing) experimental context, and this state might in principle be chosen as one of a set of basic (time dependent) vectors spanning the Hilbert space of the system. This is an interpretation of what is often called the Schrödinger picture of the system. It is of interest from the speculative point of view, but it is not of much practical use. We are left with the view expressed above that the pure case is primarily a mathematical instrument of a higher logical order capable of generating the set of mixtures associated with a determinate set of final 
experimental arrangements. Only when the final experimental context is chosen is the physicist in possession of a formula (namely, a mixture) which refers directly to concrete physical reality, i.e., to the verifiable behaviour of physical things. The mixture then represents essentially a correlation between a certain initial (or preparatory) experimental context and a definite final one. The peculiar properties of the wave function, e.g., its characteristic wave-like properties, is then to be explained by the way different matrices of transition probabilities (between initial and final states) are mathematically connected. Landé has shown that the law of interference of probabilities is identical with the law of unitary transformation of magic squares of transition probabilities when certain very reasonable symmetry conditions are fulfilled 1. While we do not wish to pass judgement on the epistemological assumptions behind Lande's polemic against the Copenhagen School, we think that his search for a realistic interpretation of quantum mechanics is a reasonable one, and that his proof that the wave function need be no more than a logical instrument connecting an ensemble of mixtures (or an ensemble of "unit magic square probability tables") is an important contribution to the epistemology of quantum mechanics.

\section{SECTION IV: FORMAL OBJECTIVITY}

\section{Formal and Public Obiectivity}

Public objectivity and formal objectivity differ in this respect, that while the former is defined by public understanding, use and definition, the latter is concerned with the part of the public object which is affirmed to belong to the domain of being. This supposes a rather different kind of analysis of the scientific object from that which merely vindicates an object of knowledge from the subjectivity of private and incommunicable experience; for even Kant could assume that people could agree in thought, language, experience and scientific principles without implying that the objects which they affirmed were objects in the formal sense, i.e., belonging to the transcendent domain of being, and he sought the a priori conditions of possibility of the public object among the synthetic pure a priori principles which human sensibility and understanding must possess. Public objects, then, in the

1 A. Lande, From Dualism to Unity in Quantum Physics (Cambridge: Cambro Univ. Press, 1960), pp. 41-54. 
Kantian explanation, were subjective vis-à-vis the formal objectivity of being except that they retained a symbolic vestige of noumenal reality through their correlation with a noumenal object which, however, was otherwise unknowable.

The first stage in the treatment of formal objectivity in science will be to distinguish in the public object what belongs properly to the content of that object, and what belongs to the method of expressing this content, characteristic of the human scientific way of knowing. For it is only the content to which formal objectivity is attributed; while public objectivity may include both the content and the method of expressing it without too much discrimination provided a common understanding is achieved. It is not surprising then that the distinction between formal object and scientific method has been largely overlooked. The failure, however, to draw a correct line between method and object in human science leads, as we have seen, to perplexing problems in quantum mechanics, not merely on the philosophic level of interpretation but on the scientific level of public objectivity.

How is the strict object of a scientific statement to be disentangled from its public object? The public object is whatever can be commonly understood and correctly used by alL The strict object is the content of a critical scientific assertion, based upon the evidence of a process of experimental testing and verification: "Such and such is so". The content of the strict object is denoted by such and such; the criterion of its formal objectivity is the scientific evidence leading to the strong use of the verb to be; viz., "is so". The assertion may be a factual assertion or a theoretical assertion. If a theoretical assertion, we take it to be converted into a set of hypothetical factual statements of the type: "If appropriate data are given, then such and such is so".

The formal objectivity of a well-tested critical scientific statement can be contrasted with its correlate which is the subjectivity of a mere supposition or hypothesis. It can be contrasted also with the public but not formal objectivity of, say, a mythic explanation, which is an explanation where symbol and reality, method and object are not separated but indissolubly united. Formal objectivity is acquired only progressively by a theory as consciousness of its epistemological structure and evidence in its favour accumulate, until something approaching a definitive judgement can be made of its validity within a well-defined domain delineated by operational and observational concepts. The ultimate goal of scientific activity is the making of such definite, unconditional, and final judgements. "In the realm of the 
exact sciences", Heisenberg wrote, "there have always been final solutions for certain limited domains of experience... The word 'final' ... means that there are always self-contained, mathematically representable, systems of concepts and laws applicable to certain realms of experience, in which realms they are always valid for the entire cosmos and cannot be changed or improved" 1.

The success, however, of this epistemological analysis of content and method is not a necessary condition of scientific progress, as the Kantian experiment has shown; for science can continue to make new discoveries and new syntheses, even when method and object are inextricably linked in the scientist's mind, and even if the meaning of "existence", "being" and "reality" are very much in doubt.

In this section, we shall try merely to separate what is affirmed viz., the content of the strict object in quantum mechanics, from what belongs to the human way of scientific knowing and so is not affirmed. We shall leave to another chapter the ontological problem of establishing the conditions under which what is correctly affirmed is truly being. We are concerned now merely with the physiognomy of the strict quantum object as possibly being.

\section{W ave-Particle Duality Re-interpreted}

In attacking psycho-physical parallelism for giving a false explanation of scientific method, we implied also a rejection on our part of the wave-particle duality of complementarity, which is a consequence of this view of scientific knowledge.

In rejecting the wave-particle duality of complementarity, we do not, however, wish to imply that some of the mathematical formulae should be changed or that observational results are not adequately accounted for by the theory. There may be inadequacies in the quantum theory, but we are not concerned with them here 2. Nor do we wish to imply that wave and particle models are useless, either as supports of our thinking or as symbolic images or as heuristic clues to further investigation. The witness of a generation of physicists proves how useful these are. Nor do we imply finally that wave-particle duality should be dropped in favour of anyone of the following: (a) a unitary "matter-particle" theory, as Popper, Bopp, Feynes,

1 Heisenberg, Physicist's Conception oj Nature, pp. 26-27 (italics our own).

2 We have already noted above (chap. v, p. 96) that many physicists, as, for example, Wigner, Ludwig, Bohm, etc., purpose to change the form of the equations of quantum mechanics, for reasons connected with the basic epistemology of the "orthodox" interpretation. 
Vigier and Landé have proposed 1; (b) a unitary "matter-wave" theory like Schrödinger's 2; or (c) a classical combination of "matterwaves" and "matter-particles" like de Broglie or Bohm have proposed 3. All of these Heisenberg has explicitly rejected because motivated by what he calls the "ontology of materialism" characteristic of classical physics 4. By a "matter-particle" we mean a bodily reality, possessing at every instant a unique set of space and momentum coordinates - whether or not these are actually and simultaneously determinable; it is then a strictly localised reality. By a "matter-wave" we mean an extended medium whose properties obey a wave equation, and vary continuously and determinately in time whether or not the properties are simultaneously and actually determinable. It is then a non-localised bodily reality. These correspond to the two separate "pictures" of complementarity and belong to co-ordinate (three-dimensional) space. There is another kind of wave, called a wave function, which is not a bodily wave, but a mathematical function on a configuration space of $3 n$-dimensions - where $n$ is the number of particles 5. This latter wave does not possess bodily objectivity, but does possess the public objectivity of a scientific object.

Our view is that the "matter-particle" and the "matter-wave" are merely real or possible observable symbols of the strict objects of physics. They are the sensible sign of the strict object, and they serve the double purpose: (I) of being the criterion for us of the reality of the strict object, since through the observable symbol the physical object

1 K. Popper, The Logic of Scientilic Discovery, (London: 1959), chap. IX; Observation and Interpretation, ed. S. Korner, pp. 65-70; F. Bopp, Werner Heisenberg usw., pp. 128-149; Ann. l'Inst. H. Poincaré, tm. xv (1956), pp. 8I-II2; J. P. Vigier, Observation and Interpretation, pp. 71-77; 1. Feynes, Zeit. f. Physik, CXXXII (1952), p. 81.

2 E. Schrödinger, "Are there Quantum Jumps?", Br. Jour. Phil. Sci., III (1952), pp. 109, 233 .

3 L. de Broglie, Non-Linear Wave Mechanics (Amsterdam: 1960); D. Bohm, Causality and Chance in Modern Physics (New York: 1957) pp. 68-128; Observation and Interpretation, $\mathrm{Pp} \cdot 33-40$.

4 Heisenberg uses the term materialism loosely. In the first place, he pays scant attention to the principal philosophic implication of the term, which is the exclusion of spirit in any but a subordinate role to matter. His thought is concerned principally with mechanistic explanations of nature. Cf. Heisenberg, Physicist's Conception of Nature, pp. 13-15. In the second place, he confuses dialectical materialism with mechanistic materialism and assumes wrongly that they share the same outlook on nature. Dialectical materialism, while not mechanistic, is opposed to Heisenberg's epistemology principally because it is a form of realism which lays claim to know physical reality objectively, i.e., in the context, to know it as it is in itself independently of the private subjectivity of the scientist-observer. Cf., Heisenberg, Niels Bohr etc., pp. 21-22; G. Wetter, Philosophie und Wissenschalt in der Sowjetunion (Munich: Rowohlts deutsche Enzyklopadie 67, 1958), pp. 7-38 and especially p. 37 where Heisenberg is criticised on this latter point.

5 Heịsenberg, Physical Principles etc., Preface. 
enters our World of reality; and (2) of yielding a measure-number for the strict object, in so far as the content of the observable symbol is a numbered content. Contrariety of symbol, however, does not imply contrariety of the essential object symbolised. The consistency and formal unity of the atomic system is expressed by a non-contradictory mathematical theory. Heisenberg was aware of this, and used it to justify the paradoxical character of complementary pictures 1. Our view in practice vis-à-vis the indeterminacy of wave and particle symbolic representations differs then very little in practice from that of Heisenberg and other complementarity physicists, but we attribute a very different epistemological significance to it.

\section{Various Unitary Re-interpretations}

Before summarising our view of the content of the strict object of quantum mechanics, we shall consider some recent opinions which try to dissolve wave-particle duality into a unitary particle theory. Bopp has proposed that the quantum mechanical object is a matter-particle with six determinate phase-space coordinates, viz., three of momentum (P) and three of position (q), only half of which at any instant are detenninable 2. This led him to experiment with various forms of non-classical statistical mechanics. The kind of object he proposed for such a theory was a virtual ensemble of "determinate but halfdeterminable" classical particles. He did not fully succeed. He was able, however, to prove a theorem of great value and interest which reads: "Any quantum mechanical system, pure and mixed states included, can be mapped into a statistical ensemble of particles in a certain phase-space: and hence also, every quantum mechanical process can be correlated to a movement of this ensemble" 3. The conclusion of his research, however, was that his "determinate but half-determinable" phase-space was not the phase-space of quantum mechanics.

The reason for this negative conclusion is interesting; for, in some respects, Bopp's theory is a test case of Proposition (1) on scientific method 4, which is a characteristic of the early view of complementarity as it was proposed and defended by Bohr and Heisenberg.

1 Cf. supra, chap. II, p. 37.

2 Bopp, Werner Heisenberg usw., loc. cit.; Observation and Interpretation, pp. 189-196; Ann. l'Inst. H. Poincaré, xv (1956), pp. 81-II2. Cf., Heisenberg's remarks in Niels Bohr etc., pp. 19-20.

3 Bopp. Observation and Interpretation, loc. cit.

4 Cf., supra, chap. IV, p. 57. 
It will be recalled that this states that quantum mechanical variables are defined through the use of classical concepts. Bopp's theory applies this principle, to which he adds the restriction typical of the quantum theory, viz., that the classical variables should be determinable only within the limits imposed by the Indeterminacy Relations. Bopp's theory is a serious attempt to translate one aspect of the epistemology of complementarity (as explained by Bohr and Heisenberg) into a physical theory. A confirmation of this can be found in the fact Heisenberg criticised Bopp's approach merely because it lacked the symmetry between wave and particle which an "orthodox" quantum theory must have and not because this modified return to classical assumptions was contrary to the quantum theory. The failure of Bopp's theory was, then, a failure of one aspect of complementarity, that which links the definition of quantum mechanical variables to classical concepts.

Our own rejection of Bopp's assumptions follows clearly from the distinction we have made between operational concepts based upon experimental techniques, and explanatory concepts based upon the understanding of a set of interrelated processes. A physical property is expressed by us as a union of both types of concepts. Position and momentum share the same operational description in classical and in quantum mechanics, but they have different explanatory definitions. The non-commutation of position and momentum operators introduces an essentially new element in the explanatory, definition of both: hence, the $\mathrm{p}$ and $\mathrm{q}$ of quantum mechanics mean something different from the $\mathrm{p}$ and $\mathrm{q}$ of classical mechanics 1 . Since the meanings are evidently related, and merge in fact in the limit of $h=0$, we can call them analogous meanings 2. To assume, as Bopp does, that they mean the same univocally but that in the quantum mechanical case they are observationally indeterminate is to misunderstand the logic of scientific method. It is the same misunderstanding which leads to the perturbation theory of measurement. It is contrary to our intention, however, to minimise the value of Bopp's undertaking which has received

1 This point has been stressed by N. R. Hanson in Patterns of Discovery (Cambridge : 1958), chap. VI and in his Concept of the Positron (Cambridge: 1963), chaps. IV-VIII; also by W. Büchel, in "Die Diskussion um die Interpretation der Quantenphysik", Scholastik, XxIX (1954) pp. 235-244, and in "Individualität und Wechselwirkung im Bereich des materiellen Seins", Scholastik, xxxi (1956), pp. 1-30.

2 There are three kinds of analogy: metaphor, metonymy or extrinsic attribution, and intrinsic proportionality; d., F. Selvaggi, "Le rôle de l'analogie dans les theories physiques", in Actes du XIe Congrès Internat. de Philos.. VI (Amsterdam-Louvain: 1953), pp. 138-143. The analogy in question in the text is most likely that of intrinsic proportionality. 
Heisenberg's praise 1, but in our view its principal value is that it has tested, with what results we have seen, one of the basic theses of complementarity 2 .

Lande, though he speaks of a unitary particle theory, does not make the mistake of defining $\mathrm{p}$ and q classically 3 . He accepts the quantum mechanical definition by non-commuting operators, and confines his re-interpretation to a statistical explanation of the wave function. For this reason, we are inclined to think that Lande's work is more acceptable than Bopp's because it is consistent with the present form of the quantum theory.

\section{The Strict Object of Quantum Mechanics}

We shall gather together here much that we have already said relevant to the strict object of quantum mechanics. It is our view that quantum mechanics is a new kind of physical theory in which both deterministic and statistical elements are organically and inseparably united 4.

From our general analysis of scientific method, we derive the proposition that the object in the strict sense of a physical theory is not per se representable in sensibility, and affects sensibility only per accidens through the presentation of an observable symbol 5. A thing or property is per se representable in sensibility if, as an object of knowledge, it is essentially constituted as the subject of typical appearances or as the typical instrument for the performance by a human subject of a certain kind of external function or for the fulfilment of a human desire. A body in the strict sense, for example, and the properties of bodies are per se representable in sensibility. An

1 Heisenberg writes that Bopp's theory "throws light upon the interesting relation between quantum theory and correlation statistics", Niels Bohr etc., p. 20.

2 We have based our argument for the essential irreducibility of quantum mechanical systems upon the results of Bopp's research rather than upon the more famous theorem of von Neumann (Mathematical Foundations etc., pp. 295-313) proving the non-existence of dispersion-free ensembles in quantum mechanics, for Bopp's "determinate but half-determinable" ensemble fulfills the conditions of von Neumann's theorem, but is still conceived to be a classical, determinate ensemble. It seems, then, that von Neumann's theorem, in spite of a widespread opinion to the contrary, does not exclude the kind of "hidden variables" conceived by Bopp. For this reason, we consider the results of Bopp's research more fundamental.

3 A. Lande, From Dualism to Unity in Quantum Physics, (Cambridge: 1960).

4 The statistical elements in question refer to what in classical physics would be called a "distribution of errors".

5 Mach, Rankine and others divided physical theories into abstractive theories dealing with perceptible objects, as for example, Newtonian mechanics; and hypothetical theories which used imperceptible hypothetical constructs, like atoms, electrons. etc., We hold this distinction to be unfounded. 
object of human scientific knowledge which is not per se representable in sensibility is per accidens representable in sensation, as, for example, through its observable symbol.

The proposition stated in the preceding paragraph applies both to physical properties and to the subjects of these properties, viz., to things. A physical property is the ground for a certain kind of interactivity between things. Hence it founds a symmetrical thing-to-thing relation. The human way of discerning the presence of this activity, then, is to study to recognise its effects on other things; these effects when transformed, magnified or otherwise processed by instrumental arrangements are the observable symbols in which the physical property is observed. It is not excluded that a physical property may be also per se representable in sensibility, but it would be difficult to establish an exact correlation between a unique sensible effect and one of the well-defined properties of physics. Usually the types of sensation correlated with physical properties are vague and composite. "The objective [scientific] concept of temperature", Weyl wrote, "is pretty far removed from the sense data of heat perception" 1 . The sense data, on the one hand, ground an observational or operational concept. The property studied in physics, on the other hand, is a thing-to-thing relation founded upon a self-correlated set of physical processes, and so is expressed by an explanatory concept.

If physical processes are not per se representable in sensibility, then neither is it necessary that the subjects which possess them should be per se representable in sensibility. A subject of physical properties is the common term of a set of relations founded upon interactivities between subjects. There is nothing in this definition which states whether such a subject is representable in sensation; and, if so, whether this is per se or per accidens. That the subject and its properties be somehow representable in sensibility follows from the fact that we, as human knowers, need a criterion of physical reality. As to how the subject is representable in sensation, the definition does not exclude per se representability. Macroscopic physical objects are represented in this way, but this does not belong to the definition of a physical object as the subject of physical properties, viz., as a thing.

Since the things which are quantum mechanical systems are not per se imaginable, it is not necessary to suppose that they are, or should be, representable in classical phase-space. A consequence of this is that we do not need to postulate the existence of "hidden variables" 
to explain the statistical laws of quantum mechanics. Moreover, quantum mechanics may be essentially, though not purely as we hold, a statistical theory; but we do not need to assume that it is or should be one of a classical or stochastic type.

From our analysis of quantum mechanics as a physical theory, we derive the proposition that the strict object of quantum mechanics is not an idealised formula of an individual system, but the individual and concrete instance of a physical system. We have already shown that individual and concrete instances can only be expressed as a virtual ensemble of cases. Quantum mechanics accounts both for the similarities between members of the ensemble - this is the deterministic part of the theory - and for the divergence between these members as far as this is possible- this is the statistical part of the theory. The function of quantum mechanics as a' deterministic theory is twofold: viz., to define by implicit definition the set of related processes which found the quantum physical properties, and to define the idealised formula, i.e., the wave function, to which the members of the ensemble conform more or less and by which the members are defined as members of the particular ensemble. The function of quantum mechanics as a statistical theory is to describe the way individual and concrete instances of the properties are distributed in an ensemble of similar systems, i.e., of systems characterised by the same wave function. Within the ensemble, individual instances are random and unsystematic with respect to the order of their occurrence, but their relative frequencies of occurrence cluster around a formula which is described by the statistical part of the theory. Since all quantum mechanical variables, including position and momentum, are defined by the deterministic part of quantum mechanics, we conclude that it is methodologically incorrect to try to construct a classical (whether deterministic or statistical) model of quantum mechanics.

\section{Matter-Form}

If neither the physical system nor its properties are per se representable in sensibility, what then is the structure of such an object? In the first place, the Kantian distinction between matter and form is no longer applicable; for the matter received into intuition is not incorporated intrinsically in the constitution of the strict object. The intuitive matter is part of the observable symbol, and so is extrinsic to the strict object as constituted an object of knowledge.

In the second place, a matter and form distinction, but not of $a$ 
Kantian type, is an intrinsic part of the strict object. The strict object is expressed by the content affirmed in a factual statement about an actual individual system. This content is precise and determinate only in so far as the individual system is expressed correctly in a definite state-function; the state-function, however, is an idealisation common to a virtual ensemble. The content is irreducibly indeterminate in so far as neither the future nor the past of the system can be fully reconstructed from this knowledge, but only the future and past of the virtual ensemble which the state-function concretely represents. The virtual ensemble, however, represents a precise distribution only because it too is an idealisation conceived as the limiting case of an infinite ensemble; or as an ideal norm from which large finite samples do not systematically diverge. Hence individual cases taken in their concreteness are not precisely defined or definable in quantum mechanics; and moreover the element of indeterminateness is irreducible.

A strict object with two such contradictory sets of properties cannot be a simple unity. It has a factor within it on which the similarity is grounded and it has some other factor on which the dissimilarity is grounded. Since the element of dissimilarity is irreducible to precise differences, it should be regarded rather as an openness to an indefinite multiplicity - one which is ruled by the ideal frequencies predicted by the wave function. If we call the ground of multiplicity, irreducible matter, and the ground of similarity, form, then the strict object has a matter and foml structure which is intrinsic to its structure, and which is not, moreover, of the Kantian type: We shall return to this discussion in chapter IX.

\section{Summary}

The "orthodox" account of the quantum mechanical measuringprocess insists on the presence of an inescapable "subjective element" in it and consequently in the heart of quantum mechanics as a physical science. We distinguish three kinds of objectivity: empirical objectivity, public objectivity and formal objectivity, to which correspond three kinds of objects; namely, empirical objects, public objects and objects in the strict or formal sense. Empirical objectivity is the characteristic of an object of classical physics; public objectivity is the characteristic of an object of science, and formal objectivity is the characteristic of Being as known. We defend the public objectivity of quantum mechanics, and then attempt to separate in the public physical object the elements which belong respectively to human scientific method, and 
to the content of the object in the strict sense. We show that the division between causal (or deterministic) theory and statistical theory is one of human scientific method, and that quantum mechanics is a new kind of theory in which both kinds of theories are united organically and inseparably. The reason for this is that quantum mechanics takes as its object in the strict or formal sense the individual instance of an ideal norm; that is, reality in its concrete manifestations. We infer, moreover, an intrinsic matter-form structure in the strict object of quantum mechanics. 